\title{
Three-dimensional reconstruction of oceanic mesoscale currents from surface information
}

\author{
Jordi Isern-Fontanet ${ }^{1,{ }^{*}}$, Guillaume Lapeyre ${ }^{2}$, Patrice Klein $^{3}$, Bertrand Chapron ${ }^{1}$ \\ and Matthew W. Hecht ${ }^{4}$
}

\footnotetext{
${ }^{1}$ Ifremer, Laboratoire d'Océanographie Spatiale, Plouzané, France.

${ }^{2}$ Laboratoire de Météorologie Dynamique/IPSL, ENS/CNRS, Paris, France.

${ }^{3}$ Ifremer, Laboratoire de Physique des Océans, Plouzané, France.

${ }^{4}$ Los Alamos National Laboratory, Los Alamos, New Mexico, USA.

*: Corresponding author : Jordi Isern-Fontanet, email address : Jordi.Isern@ifremer.fr
}

\begin{abstract}
:
The ability to reconstruct the three-dimensional (3D) dynamics of the ocean by an effective version of Surface Quasi-Geostrophy (eSQG) is examined. Using the fact that surface density plays an analogous role as interior potential vorticity (PV), the eSQG method consists in inverting the QG PV generated by sea-surface density only. We also make the extra assumption that sea-surface temperature (SST) anomalies fully represent surface density anomalies. This approach requires a single snapshot of SST and the setup of two parameters: the mean Brunt-Väisälä frequency and a parameter that determines the energy level at the ocean surface. The validity of this approach is tested using an Ocean General Circulation Model simulation representing the North Atlantic in winter. It is shown that the method is quite successful in reconstructing the velocity field at the ocean surface for mesoscales (between 30 and $300 \mathrm{~km}$ ). The eSQG framework can also be applied to reconstruct subsurface fields using surface information. Results show that the reconstruction of velocities and vorticity from surface fields is reasonably good for the upper $500 \mathrm{~m}$ and that the success of the method mainly depends on the quality of the SST as a proxy of the density anomaly at the base of the mixed layer. This situation happens after a mixed-layer deepening period. Therefore the ideal situation for the application of this method would be after strong wind events.
\end{abstract}

Keywords: Surface Quasi-Geostrophy; SST; 3D dynamics reconstruction. 


\section{Introduction}

[2] Ocean observing satellites now routinely provide global-scale measurements of surface variables (such as sea-surface temperature [SST] or sea-surface height [SSH] but, with the exception of observations within the visible spectrum, which penetrate a few tens of meters at best, no direct observations of subsurface fields. In situ subsurface measurements can provide direct observations of the threedimensional (3D) dynamics of the ocean but measurements are still sparse in space and time. This situation makes the 3D reconstruction of ocean dynamics from the available measurements a key problem in oceanography. The wealth of available data strongly encourages the investigation of reconstruction methods based on SSH and SST measurements. [3] Altimeters provide the cross-track geostrophic velocity with a relatively high along-track resolution. Since distances between tracks are generally large, interpolation methods are required to recover both zonal and meridional components of surface velocity vectors at a sufficient resolution in space and time. To circumvent such a limitation, other sources of satellite data can be considered. Over the last decades, and especially for the Gulf Stream region, early space-borne optical [Strong and DeRycke, 1973] as well as recent radar high-resolution observations [e.g., Chapron et al., 2005] have enabled the identification of very well delineated surface signatures of the upper ocean mesoscales $(30 \mathrm{~km}-200 \mathrm{~km})$ and sub-mesoscales $(<30 \mathrm{~km})$ that were not captured by altimetry. [4] These surface signatures often coincide with SST gradients and ocean color features. To obtain surface velocities from non-altimetric measurements, the analysis of sequences of images of tracer fields has then been proposed. Successful demonstrations dedicated to the validation and applicability of feature tracking clearly demonstrated that SST can potentially be a good proxy tracer [e.g., Emery et al., 1986]. Among different techniques, the Maximum Cross Correlation (MCC) method is by far the most widely used. Between consecutive images, local correlations (typically over a $20 \mathrm{~km}$ window) are computed to estimate shifts and the local motion. As expected, MCC methods act poorly in regions of uniform concentration of the considered scalar and shall present difficulties to estimate the velocity along the front of the scalar field [Zavialov et al., 2002]. Other methods can be invoked such as constrained optical flow methods to solve the heat conservation equation [e.g., Kelly, 1989; Vigan et al., 2000] or variational filter and interpolation techniques [Afanasyev et al., 2002]. All these techniques obviously rely on the availability of cloud-free conditions and also, on very highresolution sequences of images over short-enough time periods. This latter restriction is imposed by the lack of absolute conservation of the scalar SST field and somehow limits geographically and seasonally the regions over which velocities can be estimated. By analogy with turbulent flow studies, another family of methods based on the characterization of the geometry of advected tracers has been proposed [Turiel et al., 2005; Isern-Fontanet et al., 2007]. These methods are independent from the tracer used to recover velocities and can be applied to a single image from which velocity field is reconstructed. However, a lack of constraints based on oceanic dynamics still limits their performance. [5] Finally, new methodologies based on a more advanced understanding of the upper ocean mesoscale and submesoscale dynamics can be proposed. Indeed, very high numerical simulations with a Primitive Equation model of a nonlinear baroclinically unstable flow [Klein et al., 2008] suggest 
the decomposition of ocean dynamics into two competing modes: a surface-intensified mode driven by energetic smallscale structures and characterized by a power law of $k^{-5 / 3}$ for kinetic energy and surface density anomalies, and an interior mode close to the 3D quasi-geostrophic dynamics driven by potential vorticity (PV) distribution, and characterized by a power law of $k^{-3}$ for kinetic energy and a steeper spectrum slope for density. The existence of a surfaceintensified mode has been recently supported by a thorough analysis of altimeter data, which has revealed a SSH power law close to $k^{-11 / 3}$ instead of $k^{-5}$ predicted by quasigeostrophic turbulence [Le Traon et al., 2008]. Lapeyre and Klein [2006] examined the competition between the interior and the surface-intensified modes using quasi-geostrophic equations. One of their results was the dominance of the surface-intensified mode in upper oceanic layers (the first 500 meters). As a consequence, the dynamics in these layers could be modeled using an effective version of the Surface Quasi-Geostrophic (SQG) equations [e.g. Held et al., 1995], which represent a surface-intensified mode with constant Brunt-Väisälä frequency.

Using SQG equations, a complete determination of the surface stream-function can readily be obtained from a snapshot of the density anomaly. An additional major feature is the constraint that vertical scales are proportional to horizontal ones, which allows one to derive the subsurface stream-function from its surface value. This property means that subsurface velocities can be determined from instantaneous SST fields. This approach has been already tested in idealized numerical simulations [Lapeyre and Klein, 2006; LaCasce and Mahadevan, 2006], in situ measurements [LaCasce and Mahadevan, 2006] and remotely sensed data [Isern-Fontanet et al., 2006]. The objective of this paper is to further build on such a framework to study if an effective version of SQG equations can be used to reconstruct the 3D horizontal dynamics of the ocean upper layers and determine which are the best environmental conditions for the application of this reconstruction. Since coincident in situ data of subsurface currents and surface measurements at a high enough resolution are extremely rare, our analysis uses the output of numerical experiments performed using an Ocean General Circulation Model (OGCM). Current OGCM simulations are able to fairly represent most of the dynamics associated with mesoscale eddy activity (coherent eddies, fronts, filaments, eddy-eddy interactions). They may not represent submesoscale activity or high vertical mode turbulent dynamics because of their spatial resolution in the horizontal or vertical but we are mainly interested in the mesoscale signal and its reconstruction using surface information. Therefore the OGCM simulation is a good test field for our study.

The paper is organized as follows: section 2 introduces the theoretical background and the dynamical relations that exist between SST and SSH. Section 3 describes some details of the OGCM simulation we use and the procedure of the reconstruction. Section 4 is focused on the reconstruction of surface fields from SST and section 5 on the reconstruction of subsurface fields using SST and SSH. Finally, sections 6 and 7 discuss our results and present our conclusions.

\section{Quasi-geostrophic PV inversion}

The principle of invertibility of potential vorticity [see Hoskins et al., 1985] allows the diagnosis of the 3D dynamics of a balanced flow from the knowledge of PV in the ocean interior and density on the vertical boundaries. If we assume that the flow is in Quasi-Geostrophic (QG) equilibrium the problem consists in inverting the equation

$$
\nabla^{2} \psi+\frac{\partial}{\partial z}\left(\frac{f_{0}^{2}}{N^{2}} \frac{\partial \psi}{\partial z}\right)=Q
$$

where $Q(x, y, z)$ is the $\mathrm{PV}$ anomaly, $\psi(x, y, z)$ the streamfunction of the flow, $f_{0}$ the local Coriolis frequency, $N(z)$ the Brunt-Väisälä frequency and $\nabla$ only denotes the horizontal operator $\left(\nabla \equiv\left(\partial_{x}, \partial_{y}\right)\right)$. The appropriate boundary condition at the surface can be derived from the hydrostatic equation. Then, for a semi-infinite domain, boundary conditions are

$$
\begin{gathered}
\left.f_{0} \frac{\partial \psi}{\partial z}\right|_{z=0}=b_{s} \\
\lim _{z \rightarrow-\infty} \frac{\partial \psi}{\partial z}=0
\end{gathered}
$$

with the limit indicating our use of a right-handed coordinate system with $z$ increasing to 0 as we approach the surface. Surface buoyancy $b_{s}(x, y)$ is defined as

$$
b_{s}=-\frac{g}{\rho_{0}} \rho_{s},
$$

which is computed from the density anomaly at the surface $\rho_{s}(x, y)$ and a reference density $\rho_{0}$.

Mathematically, the inversion of equation (1) subject to the boundary conditions of equations (2) and (3) can be split into two different problems: a surface-forced solution $\psi_{s r f}(x, y, z)$ obtained by taking zero PV but keeping surface buoyancy $\left(Q=0, b_{s} \neq 0\right)$ and an interior-forced solution (or interior mode) $\psi_{\text {int }}(x, y, z)$ obtained by taking zero surface buoyancy but keeping $\operatorname{PV}\left(Q \neq 0, b_{s}=0\right)$. Then, the total solution will be

$$
\psi=\psi_{\text {srf }}+\psi_{\text {int }}
$$

Assuming a constant stratification $\left(N(z)=N_{0}\right)$ the surfaceforced problem can be written in the Fourier space as

$$
-k^{2} \widehat{\psi}_{s r f}+\frac{f_{0}^{2}}{N_{0}^{2}} \frac{\partial^{2} \widehat{\psi}_{s r f}}{\partial z^{2}}=0
$$

where $\widehat{(\cdot)}$ stands for the horizontal Fourier transform, $k=$ $\|\mathbf{k}\|$ is the modulus of the horizontal wave-vector $(\mathbf{k}=$ $\left.\left(k_{x}, k_{y}\right)\right)$. Then, in the horizontal Fourier transform domain the solution of equations (2), (3) and (6) simply reduces to

$$
\widehat{\psi}_{s r f}(\mathbf{k}, z)=\frac{\widehat{b}_{s}(\mathbf{k})}{N_{0} k} \exp \left(\frac{N_{0} k z}{f_{0}}\right) .
$$

Equation (7) is termed the Surface Quasi-Geostrophic (SQG) system [Held et al., 1995]. This solution can be viewed as a surface-trapped response (decaying exponentially with depth) to a Dirac function of PV at the ocean surface $z=0$ [Bretherton, 1966]. The horizontal and vertical scales are strongly coupled: the smaller horizontal scales decay in the vertical faster than larger ones. This is due to the condition of uniform PV anomaly $(Q=0)$ that gives equation (6).

\subsection{Link between surface and interior forced streamfunctions}

In the ocean one cannot assume that interior PV is zero so the interior PV contribution $\psi_{\text {int }}$ cannot be neglected. However, Lapeyre and Klein [2006] have recently demonstrated that, for the first 500 meters the surface-trapped solution $\psi_{\text {srf }}$ could represent the total solution $\psi$ for baroclinically unstable flows forced by a large-scale density gradient. In 
this situation, the large-scale forcings in density and PV will lead to the property that the interior PV mesoscale anomalies are correlated to the surface buoyancy anomalies. In that case, the PV anomaly can be separated as $Q \approx \phi(z) b_{s}$. This implies that $\widehat{\psi}$ can also be separated as $\widehat{\psi}_{\text {int }}(\mathbf{k}, z)=\widehat{\varphi}(k, z) \widehat{b}_{s}(\mathbf{k})$ and $\widehat{\varphi}(k, z)$ verifies

$$
-k^{2} \widehat{\varphi}+\frac{f_{0}^{2}}{N^{2}} \frac{\partial^{2} \widehat{\varphi}}{\partial z^{2}}=\phi,
$$

An important consequence is that $\psi_{s r f}$ and $\psi_{\text {int }}$ will possess correlated spatial structures (since their spectral transform are both proportional to $\widehat{b}_{s}$ ). Only their vertical variations will differ.

As Lapeyre and Klein [2006] have shown, the contribution of $\psi_{\text {int }}$ is in general smaller than $\psi_{\text {srf }}$ in the first $500 \mathrm{~m}$. This is because the surface density gives a larger contribution than the upper layer PV in terms of PV anomalies. Therefore, $\psi_{\text {int }}$ only modifies the vertical variation of the total solution $\psi$. As $\psi_{\text {int }}$ mostly depends on the barotropic and the first baroclinic modes and as these modes only vary weakly in the first 300 meters (contrary to the variation of $\psi_{\text {srf }}$ which is more important), the total solution can be approximated by an effective SQG solution (eSQG) obtained from the substitution of $N_{0}$ in the denominator of (7) by a value empirically obtained from the comparison with indpendent observations (see below).

The eSQG method will presumably not work if mesoscale eddies are intensified underneath the surface or have no surface density anomalies, so that the contribution $\psi_{\text {srf }}$ would be smaller than $\psi_{\text {int }}$.

\subsection{The eSQG model}

Introducing the Prandtl ratio $n$ [e.g. Tulloch and Smith, 2006] as

$$
n=\frac{N}{\left|f_{0}\right|}
$$

the eSQG model for the buoyancy $b(x, y, z)$ and the streamfunction $\psi(x, y, z)$ can be written as

$$
\widehat{b}(\mathbf{k}, z) \approx \widehat{b}_{s}(\mathbf{k}) \exp \left(n_{0} k z\right)
$$

and

$$
\widehat{\psi}(\mathbf{k}, z) \approx \frac{\widehat{b}_{s}(\mathbf{k})}{f_{0} n_{b} k} \exp \left(n_{0} k z\right)
$$

where $n_{b}$ is the empirically-determined effective Prandtl ratio required to take into account the contribution of the interior PV and $n_{0}$ is simply the Prandtl ratio associated to the Brunt-Väisälä frequency $\left(N_{0}=\left|f_{0}\right| n_{0}\right)$.

As derived, the eSQG model allows the reconstruction of geostrophic velocities in the upper oceanic layers from only one snapshot of the surface density field $b_{s}$ and two parameters: the Brunt-Väisälä frequency $\left(n_{0}\right)$ that, determines the vertical atenuation of surface fields and depends on the large-scale properties of the flow, and the effective Prandtl ratio $\left(n_{b}\right)$ that, determines the energy level at the ocean surface and depends on the mesoscale. In addition, the eSQG model allows the reconstruction at depth of any linear function of $\widehat{\psi}$ from its observed value at the surface, e.g.

$$
\widehat{\mathbf{v}}(\mathbf{k}, z)=\widehat{\mathbf{v}}_{s}(\mathbf{k}) \exp \left(n_{0} k z\right)
$$

and

$$
\widehat{\zeta}(\mathbf{k}, z)=\widehat{\zeta}_{s}(\mathbf{k}) \exp \left(n_{0} k z\right)
$$

where $\mathbf{v}=(u, v)$ is the horizontal velocity, $\zeta=\partial_{x} v-\partial_{y} u$ the relative vorticity and $\mathbf{v}_{s}$ and $\zeta_{s}$ their surface values. Notice that $\widehat{\mathbf{v}}=\left(-i k_{y} \widehat{\psi}, i k_{x} \widehat{\psi}\right)$ and $\widehat{\zeta}=-k^{2} \widehat{\psi}$ so that these quantities can be obtained from surface buoyancy using equation (11).

\subsection{Relationship between surface fields}

Equation (11) has important consequences on the relationships between Sea Surface Height (SSH) and Sea Surface buoyancy. The sea surface height $\eta$ provides a direct estimation of the stream-function at the surface:

$$
\psi_{s}=\frac{g}{f_{0}} \eta
$$

Then, one has a direct relation between SSH and surface buoyancy,

$$
\widehat{\eta}=\frac{1}{n_{b} g} \frac{\widehat{b}_{s}}{k} .
$$

Equations (14) and (15) imply that the surface kinetic energy spectrum has to be proportional to the buoyancy spectrum:

$$
E=k^{2}|\widehat{\psi}|^{2}=A^{2} k^{2}|\widehat{\eta}|^{2}=B^{2}\left|\widehat{b}_{s}\right|^{2}
$$

with $A=g / f_{0}$ and $B=1 / f_{0} n_{b}$.

Similar relations can be obtained using Sea Surface Temperature (SST), if we assume that salinity is for instance constant. In that case, surface buoyancy can be directly derived from SST as

$$
b_{s} \approx \frac{g \alpha}{\rho_{0}} T_{s}
$$

where $\alpha>0$ is the thermal expansion coefficient and $T_{s}$ is the temperature anomaly. In the same spirit of the eSQG equations (10) and (11), we can introduce an eSQG solution but using a different effective Prandtl ratio $n_{T}$ for the mean kinetic energy level. This parameter is different from $n_{b}$ and allows one to account for the salinity contribution (through the density anomaly). In that case, we would obtain

$$
\widehat{\eta}=\frac{\alpha}{n_{T} \rho_{0}} \frac{\widehat{T}_{s}}{k} .
$$

and

$$
E=C^{2}\left|\widehat{T}_{s}\right|^{2}
$$

with $C=g \alpha / f_{0} n_{T} \rho_{0}$.

These different relations provide a test of the pertinence of the SQG theory and could be in principle examined at a global scale using satellite data.

\section{Numerical experiments}

\subsection{Model}

To investigate the capabilities and limitations of the eSQG method, we have chosen to use the output of a realistic simulation of the North Atlantic Ocean as a test field. Using an OGCM allows for direct access to all variables at surface and at depth so that the sensitivity of the method can be assessed as a function of external parameters (mixed layer depth, etc.). The POP model at $1 / 10^{\circ}$ [Smith et al., 2000; Bryan et al., 2007] is suitable for this type of study because it is forced with realistic winds and heat fluxes and 
it resolves the mesoscales quite well. A comparison with TOPEX altimeter data has shown that it had similar characteristics in terms of energy and lengthscales [Brachet et al., 2004].

The model grid has a uniform aspect ratio, with the meridional spacing set to be equal to the zonal spacing. The zonal grid spacing is 0.1 degree, which is $11.1 \mathrm{~km}$ at the equator. The meridional grid spacing is 0.1 degrees multiplied by the cosine of latitude, in order to maintain uniform aspect ratio. The model has been forced with daily averaged ECMWF winds. It uses the vertical mixing scheme provided by the K-Profile Parametrization [Large et al., 1994]. We have used a daily average taken in January in order to filter a large part of near-inertial waves present in the velocity and vorticity field. The particular day which was analyzed (January 20, 2002) contains a strong wind forcing event, allowing us to test our methodology over a wider range of conditions.

\subsection{Procedure}

The region of the North Atlantic under study has been divided into 34 boxes of approximately $650 \times 550 \mathrm{~km}$. The fields obtained in each box from the numerical experiment have been projected onto a plane using a Mercator projection. Each box consists in a minimum of $60 \times 60$ and a maximum of $60 \times 72$ grid points. Projected fields have been reinterpolated onto a doubly-periodic Fourier grid of $128 \times 128$ grid points using mirror symmetry to obtain periodic fields in $x$ and $y$. Finally, their Fourier transforms have been computed. If a sea-mount or an island is present at a particular grid point in $x, y$ space, the missing data is replaced by a weighted average of valid points around it. Weights are proportional to the inverse of the distance.

\subsection{Main characteristics}

Figure 1 shows the surface vorticity field corresponding to January 20, 2002. For each box the RMS value of surface relative vorticity, normalized by $f_{0}\left(\right.$ i.e. $\left\langle\zeta^{2}\right\rangle^{1 / 2}\left|f_{0}\right|^{-1}$ ), has been found to be within the range 0.03 and 0.3 . Interpreting it as a mean Rossby number and as indicator of the eddy activity, this points out the presence of energetic eddies and weak to moderate ageostrophic processes, specially in those boxes dominated by the presence of the meanders of the Gulf Stream (boxes 5, 9, 10 and 14 have values of the order of 0.25$)$.

The maximum wind stress observed in the preceding 2 days $\left(\tau_{m}\right)$ has been estimated from QuikSCAT Level 4 Gridded Mean Wind Fields (MWF-QuikSCAT product $0.5 \times 0.5$ degree) downloaded from CERSAT data center (cersat.ifremer.fr). This two day period is assumed to affect the mixed layer evolution. The spatial distribution of $\tau_{m}$ (see figure 1) is inhomogeneous with the most intense winds located to the northeast part of the analyzed region, decreasing towards the southwest. Winds in the region of the Azores and Portugal currents were very weak during the previous two days. The RMS of the surface wind stress $\left(\left\langle\tau_{m}^{2}\right\rangle^{1 / 2}\right)$ ranges from values close to 0 in boxes around box 42 to values close to $0.8 \mathrm{~Pa}$ for boxes 36 and 40 .

The mixed layer depth $\left(h_{m l}\right.$, figure 1$)$ has been estimated as the shallowest depth at which potential density differs from the density at $5 \mathrm{~m}$ by at least $0.07 \mathrm{~kg} \mathrm{~m}^{-3}$ [see de Boyer Montégut et al., 2004, and references therein]. Observed RMS were of the order of $100 \mathrm{~m}$ ranging from $85 \mathrm{~m}$ in the region of the East Azores and Portugal currents (boxes around $38,41,42,46,47)$ to $150 \mathrm{~m}$ in the region with the strongest winds (boxes 35, 36, 40, 44 and 48). In the southwestern part of the basin the depth of the mixed layer was observed to be around $120 \mathrm{~m}$ (boxes 5 and 9). Boxes 19, 23 and 24 are partly located over the continental shelf and have very shallow mixed layers covering a significant part of the box.
The alignment and compensation in density between salinity and temperature gradients have been measured using the complex ratio given by Ferrari and Paparella [2003]

$$
r=-\frac{\alpha}{\beta} \frac{\partial_{x} T+i \partial_{y} T}{\partial_{x} S+i \partial_{y} S}
$$

where $T$ and $S$ are temperature and salinity anomalies (as in section 2.3), $\alpha>0$ is the thermal expansion coefficient, $\beta>0$ is the expansion coefficient of salinity and $i=\sqrt{-1}$. The phase $\theta_{r}$ of the complex ratio $r$ quantifies the degree of alignment of salinity and temperature gradients and the magnitude of their relative strength. Then, thermohaline compensation (cancellation of temperature gradients by salinity gradients in density) corresponds to $|r|=1$ and $\theta_{r}=180^{\circ}$. The mean complex ratio $(\langle r\rangle)$ within each box has been computed for the mesoscale (wavelengths smaller than $350 \mathrm{~km}$ ) at two different depths: within the mixed layer $(5 \mathrm{~m})$ and below it $(268.5 \mathrm{~m})$. In general, there is a strong alignment between salinity and temperature gradients with a mean phase over the boxes of $176.95^{\circ} \pm 3.13^{\circ}$ within the mixed layer ${ }^{1}$ and $178.83^{\circ} \pm 0.96^{\circ}$ below it. The magnitude of $\langle r\rangle$ shows a mean value over all boxes of $1.41 \pm 0.61$ within the mixed layer and $2.47 \pm 0.49$ below it. This indicates that in general there is a dominance of temperature.

\subsection{Parameter adjustment}

The attenuation parameter $n_{0}$ is involed in the uniform potential vorticity equation (6). We therefore choose it to be equal to $N_{0} / f_{0}$ with $N_{0}$ an averaged Brunt-Väisälä frequency in the first $1000 \mathrm{~m}$ (see table 1). Observed values range from 20 in the northeastern part of the domain to 60 in the southwestern region.

To estimate the parameters $n_{b}$ and $n_{T}$ that set the mean kinetic energy level, it is necessary to have additional information. Since our objective is to develop a general methodology for the reconstruction of ocean dynamics from observations, we have assumed that we have access to high resolution SST or surface density ${ }^{2}$ and low resolution SSH. This situation would correspond to having infrared SST images and SSH maps derived from current altimetric measurements.

In such a situation, we consider a band-pass filtered SSH using cut-off wavelengths of $100 \mathrm{~km}$ and $350 \mathrm{~km}$. The resulting low-resolution SSH $\left(\eta_{L}\right)$ are then used to estimate surface mesoscale kinetic energy: $E_{\eta_{L}}=g^{2}\left|\nabla \eta_{L}\right|^{2} / f_{0}^{2}$. Then surface buoyancy $b_{s}$ and sea surface temperature $T$ are bandpass filtered to produce surface fields $b_{s L}$ and $T_{L}$ at the same spatial resolution as the low-resolution SSH. Using equations (16), the Prandtl ration $n_{b}$ can be obtained as

$$
n_{b}=\frac{1}{f_{0}} \sqrt{\frac{\left\langle b_{L}^{2}\right\rangle}{\left\langle E_{\eta_{L}}\right\rangle}} .
$$

Using equation (18) gives

$$
n_{T}=\frac{g \alpha}{f_{0} \rho_{0}} \sqrt{\frac{\left\langle T_{L}^{2}\right\rangle}{\left\langle E_{\eta_{L}}\right\rangle}}
$$

Table 1 shows the values of $n_{b}$ and $n_{T}$. An initial comparison between $n_{0}, n_{b}$ and $n_{T}$ reveals that the magnitude of $n_{b}$ is generally quite close to $n_{0}$, while the magnitude of $n_{T}$ is larger than the values of $n_{0}$ and $n_{b}$. This confirms that the compensation between temperature and salinity is important (see section 3.3) and that the salinity distribution has a stronger effect on the magnitude of surface fields than the interior PV. 


\section{Surface flow reconstruction}

To illustrate in more detail the capabilities of the reconstruction based on the eSQG equations, four boxes characterized by good reconstruction results have been selected. Three of these boxes $(26,32$ and 40$)$ are located in a region of the North Atlantic Drift of moderate to strong winds, in which SST and surface buoyancy should be a priori good proxies of buoyancy below the mixed layer. The fourth box is located in an area of low wind stress but deep mixed layer in the Western part of the Sargasso sea (box 13).

\subsection{Spectral comparisons between surface fields}

Equations (16) and (19) predict the same spectra for surface geostrophic kinetic energy and surface potential energy provided that they are properly dimensionalized. To test this prediction, we have computed $E_{\eta}=A^{2} k^{2}|\widehat{\eta}|^{2}$, $E_{b}=B^{2}\left|\widehat{b}_{s}\right|^{2}$ and $E_{T}=C^{2}\left|\widehat{T}_{s}\right|^{2}$. To compare directly the spectral slopes of each spectrum, we have rescaled $E_{b}$ and $E_{T}$ so that $E_{\eta}\left(k_{0}\right)=E_{b}\left(k_{0}\right)=E_{T}\left(k_{0}\right)$ for a wavenumber $k_{0}=10^{-4} \mathrm{~m}^{-1}$. For the largest observed wavelengths, $E_{b}$ and $E_{T}$ contain more energy than $E_{\eta}$, as evident from the energy spectra of boxes 13,26, 32 and 40 shown in figure 2 . These larger wavelengths are obviously not related to oceanic currents but to the large-scale density or temperature forcing. For wavelengths below $300 \mathrm{~km}\left(k \simeq 2 \times 10^{-5}\right.$ $\mathrm{m}^{-1}$ ) spectral slopes are very similar. Indeed, between 31 $\mathrm{km}$ and $214 \mathrm{~km}\left(3 \times 10^{-5} \leq k \leq 2 \times 10^{-4}\right)$ the mean slopes over all boxes are $-3.8 \pm 0.3,-3.9 \pm 0.4$ and $-3.8 \pm 0.3$ for $E_{b}, E_{T}$ and $E_{\eta}$ respectively. Boxes 19 and 24 have been excluded from this estimation due to the poor eSQG reconstruction results (see below).

A close scrutiny of the spectral relationships between surface fields is provided by the calculation of the spectral correlations between surface vorticity $\left(\zeta_{s}=\zeta(z=0)\right)$ and its prediction from SSH $\left(\widehat{\zeta}_{\eta}(z=0)=-g k^{2} \widehat{\eta} / f_{0}\right.$ using equation $(14))$, or from surface buoyancy $\left(\widehat{\zeta}_{b}(z=0)=-k \widehat{b}_{s} / f_{0} n_{b}\right)$ or from $\operatorname{SST}\left(\widehat{\zeta}_{T}(z=0)=-\alpha g k \widehat{T}_{s} / f_{0} \rho_{0} n_{T}\right.$ using equations (11) and (17)). To this end, the spectral range has been divided into 60 bins and for each bin the correlation between the real parts of the variables has been computed. Results show that the best correlations are found between $\zeta_{s}$ and $\zeta_{\eta}(z=0)$ at, least for length scales between 60 and $350 \mathrm{~km}$, where correlations are of the order of 0.9 for almost all boxes (not shown). In box 5, which is the box with the highest Rossby number, spectral correlations are of the order of 0.8 in the whole range. However, in many boxes, such as the ones presented in figure 2, high correlations span almost the entire observable range. This high correlation indicates that the surface velocity field is close to geostrophic balance.

Spectral correlations between $\zeta_{s}$ and $\zeta_{b}(z=0)$ or $\zeta_{T}(z=$ $0)$ enable one to test the applicability of the eSQG approach. For the upper range of mesoscales (scales between 80 and $200 \mathrm{~km})$ the mean correlation between $\zeta_{s}$ and $\zeta_{b}(z=0)$ (respectively $\zeta_{T}(z=0)$ ) is around 0.67 (respectively 0.68 ). This suggests that in some boxes the eSQG method using surface buoyancy provides a better estimate of vorticity while in some other boxes the best estimations are obtained using SST. This is confirmed by the visual inspection of the spectral correlations in all boxes. A closer look at the spectral correlations reveals that in some cases (e.g. box 26 in figure 2) the correlation between $\zeta_{s}$ and $\zeta_{b}(z=0)$ drops at wavelengths between 50 and $150 \mathrm{~km}$. On the other hand, surface buoyancy provides a better estimation of the vorticity field than SST at scales between 30 and $60 \mathrm{~km}$ $\left(10^{-4} \leq k \leq 2 \times 10^{-4} \mathrm{~m}^{-1}\right)$, with correlations which oscillate around 0.73 , as compared with correlations of around 0.63 for $\zeta_{T}(z=0)$ (e.g. box 32 in figure 2). We do not have an explaination of this phenomenon. This might be linked to processes affecting the mixed layer (see below) or a deficiency of the simulation at these scales and in this region.

\subsection{Pattern reconstruction}

The surface stream-function in each box has been obtained from surface buoyancy and SST using equations (11) and (17), applying a high-pass filter with a wavelength cutoff of $350 \mathrm{~km}$. From these stream-functions, surface velocity and vorticity have been computed. A first inspection of the reconstructions shown in figure 3 reveals that surface velocity and vorticity computed from SSH tend to be almost identical to the model velocity and vorticity. Some differences may exist for the strongest currents (the cyclonic structure near $x=70 \mathrm{~km}$ and $y=500 \mathrm{~km}$ in box 26 , or the anti-cyclonic structure at $x=100 \mathrm{~km}$ and $y=100 \mathrm{~km}$ in box 32 for instance). Comparing the vorticity and velocity fields obtained from the surface buoyancy reconstruction and from SSH (figure 3), we see a good correspondence between the fields. Some noticeable differences are visible. The eSQG reconstruction using buoyancy tend to intensify too much the vorticity and kinetic energy compared to observed geostrophic velocity and kinetic energy in regions of sharp density fronts (see for instance the small-scale filament that detach from an eddy at $x=350 \mathrm{~km}$ and $y=330 \mathrm{~km}$ in box 32 ). In box 26 , the vorticity sign within the vortex located at $x=50 \mathrm{~km}$ and $y=500 \mathrm{~km}$ is reversed, which coincides with the small spectral correlations observed between 50 and $150 \mathrm{~km}$ (figure 2). The inspection of all boxes reveals that this phenomenon can be found in other boxes such as 22,23 , 27, 28 and 31 (not shown). The vorticity and velocity fields obtained from SST resemble the model fields. It is interesting to note that the reconstruction well behaves for the cyclone at $x=50 \mathrm{~km}$ and $y=500 \mathrm{~km}$ in box 26 , contrary to the buoyancy reconstruction.

The scatter plots of velocity and vorticity have been computed for boxes 13, 26, 32 and 40. The range of model velocities and vorticities has been divided into 100 bins and the mean value of the corresponding eSQG field has been calculated. Figure 4 shows the scatter plots for these boxes. These plots give another confirmation of the good reconstruction capabilities of the eSQG model. For large values of vorticity, the data scatter from the bisector, which means that the reconstruction does not work when currents become strongly ageostrophic.

To quantify the quality of the reconstruction the linear correlation coefficients between the reconstructed vorticities from SSH, surface buoyancy and SST and vorticity derived from the model velocities have been computed for each box (figure 5). Linear correlations between surface velocities (respectively vorticity) estimated from SSH and from the model provide the highest correlations with values 0.93 (respectively 0.98) (not shown). Reconstructions of vorticity from surface density range between 0.65 to 0.9 correlations with two exceptions: the North Atlantic Drift (boxes 19, 22, 23, 24, 26, 27, 28 and 31) and the region of the East Azores and Portugal currents (boxes 38, 41, 42, 43, 46 and 47). Surface correlations between SST-derived and model fields exhibit similar patterns although some main differences are evident. On one side, the North Atlantic Drift (boxes 22, 23, $26,27,28$ and 31) presents higher values of correlation for SST-derived fields. On the other side, in the region south of Azores islands (boxes 25, 29, 33, 37) buoyancy-derived fields have higher correlations. Furthermore, a global look at surface correlations shows that SST provides better correlations than surface buoyancy in 15 boxes $(19,22,23,26$, 27, 28 and 31 are clearly better and 9,24,36, 41 and 43 slightly better), while 10 boxes may be found in which the situation is the opposite $(10,25,29,33,34$ and 37 are clearly worse and 14, 38, 39, 46 are slightly worse).

Concerning surface velocities, the reconstruction from SST seems to be more accurate than the reconstruction from 
surface density, particularly in the area of influence of the Gulf Stream and the North Atlantic Drift (boxes 19, 22, 23 , 26, 27, 28 and 31). This is evident in the case of meridional velocities, where correlations of the order of 0.8 can be observed in this area. Although in zonal velocities this result is less evident (see boxes 10,14,19), it is still possible to observe a similar pattern.

These results demonstrate that the eSQG approach gives quite good reconstruction of horizontal velocity and relative vorticity at the ocean surface for most of the boxes in the North Atlantic. However, surface correlations strongly vary from one area to the other. An important question is thus to identify which external parameters (wind, mesoscale activity...) a good reconstruction depends on. To simplify the problem, we first focused on the reconstruction of vorticity from SST. Correlations of model vorticity with reconstruction have been compared to the RMS of surface vorticity $\left(\left\langle\zeta^{2}\right\rangle^{1 / 2}\right)$, the RMS of the maximum wind stress observed during the preceding two days $\left(\left\langle\tau_{m}^{2}\right\rangle^{1 / 2}\right)$ and the RMS of the mixed layer depth $\left(\left\langle h_{m l}^{2}\right\rangle^{1 / 2}\right)$. Figure 6 shows that there is a tendency for increasing correlation with increasing vorticity RMS for $\left\langle\zeta^{2}\right\rangle^{1 / 2}<0.15 f_{0}$. Indeed, computing the correlation between the two fields gives a value of 0.40 , but if only those boxes with $\left\langle\zeta^{2}\right\rangle^{1 / 2}<0.15 f_{0}$ are compared, it increases to 0.70 . This means that when the eddy activity increases, the reconstruction performs better. However, if vorticity is too high, ageostrophic processes become important, the QG assumption becomes invalid, and the correlation decreases. We indeed observed a decrease of the correlation between vorticity and its reconstruction from $\mathrm{SSH}$ when enstrophy increases, which tends to confirm the negative effect of ageostrophic processes associated with high enstrophy values.

Although the RMS of vorticity is indicative of different dynamical regimes, the characteristics of the mixed layer have a deep impact on the SST and therefore, on the reconstruction capabilities of the eSQG approach. The scatter plot between the correlation and the RMS of the wind stress shows that the correlation increases with increasing winds. However, in three boxes located in the Sargasso Sea (boxes 9,13 and 17) the reconstruction is good even though the winds are weak. The correlation with wind RMS is 0.55 . If boxes 9, 13 and 17 are eliminated it increases to 0.79 . The scatter plot between correlations and the RMS of the mixed layer depth clearly shows that deeper mixed layers are associated with better reconstruction. The correlation with mixed layer depth RMS is 0.73 . If box 28 is eliminated it increases to 0.84 . A similar study was performed with an eSQG approach using surface buoyancy instead of SST but the results were much less clear. This suggests that density does not respond in the same manner as SST. We hypothesize that this is related to a strong relationship between salinity and temperature anomalies in the mixed layer. This will be discussed in Section 6 .

Finally, interior PV anomalies may also impact the reconstruction. Lapeyre [2008] has considered the same simulation and has decomposed the vorticity field into a part associated with the interior PV field (but with no surface buoyancy) and a part associated with the surface buoyancy field (but with no interior PV). Using his data, we have compared the correlation of the SST reconstruction and the observed field with the ratio of RMS of vorticity due to surface buoyancy and the RMS of vorticity due to interior PV. We found indeed a positive correlation of 0.64 between the two fields which indicates that the eSQG reconstruction performs better when the contribution of the interior PV in the inversion is smaller. However this is still smaller than the correlation obtained with the wind, so this effect is less important than the variability of the Mixed Layer.

\section{Reconstruction on the vertical}

\subsection{Spectral correlations at depth}

A major output of the eSQG approach is not only the recovery of surface dynamics from surface density or tem- perature, but also the recovery of currents at depth. Indeed, the eSQG equations (10), (11), (12) and (13) predict that deep fields should resemble surface fields but multiplied by $\exp \left(n_{0} k z\right)$. This function acts as a low-pass filter of the surface fields since for a given $z$ it damps small scales. Therefore, correlations between surface fields and deep fields are expected to depend on the wavelength. Larger horizontal scales will penetrate deeper than smaller scales, so we expect the reconstruction to become invalid first at small scales when going at depth.

This result has been verified by computing the spectral correlation coefficient between vorticity at certain depths $(\widehat{\zeta}(z))$ and surface vorticity estimated from SSH (Figure 2, third row). Results reveal that for large wavelengths (small $k$ ), spectral correlations derived from $\mathrm{SSH}$ can be higher than 0.7 at depths of the order of $1000 \mathrm{~m}$. For small wavelengths (large $k$ ) high correlations are observed only close to the surface. Furthermore, wavelengths smaller than $40 \mathrm{~km}$ $\left(k \simeq 1.2 \times 10^{-4} \mathrm{~m}^{-1}\right.$ ) have correlations higher than 0.7 only within the mixed layer or slightly below.

The correlation of vorticity at depth with surface vorticity computed from SST shows similar features (Figure 2, last row), although the high correlations do not extend as deep as in the case of correlations with surface vorticity from SSH. Spectral correlations calculated from surface buoyancy present lower values at depth for the same bands for which poor correlations have been observed at surface. For wavelengths smaller than $40 \mathrm{~km}$, surface vorticity derived from surface buoyancy is higher or equally correlated to vorticity than surface vorticity derived from SST.

This result suggests that, as previously outlined, if an estimate of the surface stream-function is already available (from SSH or SST), then the eSQG approach allows for reconstruction of the dynamics at depth.

\subsection{Reconstruction at depth}

3D flows are reconstructed for the first $1000 \mathrm{~m}$, based on surface fields calculated in section 4.2. Figure 7 shows the reconstruction at $500 \mathrm{~m}$ depth. The comparison between figures 3 and 7 highlights the resemblance of deep fields to a low-passed version of surface fields. A remarkable visual coincidence is exhibited between the four fields. It is interesting to note that the filtering process associated with the vertical propagation of surface fields has eliminated the sign inversion observed in box 26, comparing therefore better with the true field. The reconstruction from SSH and SST gives patterns at the right scale, whereas reconstruction from buoyancy seems to be at larger scales (this is particularly obvious from box 32 ).

To isolate the vertical reconstruction capabilities of the eSQG framework from the quality of surface SST and buoyancy we first focus on the vertical reconstruction prediction derived from SSH. Results show that vorticity and velocity correlations decrease monotonically with depth (see the examples in figure 8). The reconstruction is quite good for the first upper 200 meters, with correlations larger than 0.9 for vorticity, and also for zonal and meridional velocities. The correlations decrease with depth but remain significant at 1000 meters. An estimate of the depth at which the fields can be reconstructed from surface fields can be obtained by selecting a threshold value for correlations, using vorticity as a test field. If a threshold value of 0.7 is chosen, then the method can reconstruct the dynamics in the upper $500 \mathrm{~m}$ of the ocean, on average. A threshold value of 0.9 reduces the depth to approximately the upper $150 \mathrm{~m}$. 
Vertical reconstructions using SST or surface buoyancy depend strongly on the quality of the surface fields which are used. Correlations also decrease with depth and are always lower than the correlations obtained from SSH (see the examples in figure 8). There are, however, some exceptions, in boxes 19, 23 and 28. The maximum correlation for the SST-derived vorticity is found within the first hundred meters of the ocean and for box 25 at depths between 400 and 500. To verify that the eSQG framework reconstructs better deep flows than the assumption of barotropic fields, the correlation between vorticity at depth $z$ and surface vorticity has been compared to the results previously obtained. In all situations the eSQG approach has higher correlations as depth increases (not shown).

Correlations of model buoyancy with reconstructions give a non-monotonic signal (top row of figure 8). For the first 200 meters, the SSH reconstruction gives the lowest correlations compared to SST and buoyancy reconstructions. By definition, buoyancy has a perfect reconstruction at the surface using $b_{s}$ in the eSQG approach. However at depth, the $\mathrm{SSH}$ reconstruction becomes superior, as the buoyancy reconstruction decreases quite rapidly (this is obvious for box 40). It is remarkable that in boxes located in the NorthAtlantic Drift (boxes 19, 22, 23, 26, 27, 28 and 31) within the mixed layer the SST-derived buoyancy has poor correlations, while below the mixed layer correlations increase and are significantly larger than the correlations obtained from surface buoyancy at the same depth. In other boxes (e.g. box 40) buoyancy correlations decrease faster when computed from $b_{s}$ than from SST, which gives higher correlations of SST-derived buoyancies below a certain depth. This result points to the existence of a mixed layer process that makes surface buoyancy deviate from its value at the base of the mixed layer, making SST resemble subsurface density. This is consistent with the low correlations found when surface fields were reconstructed from surface buoyancy and the high correlations found when they were reconstructed from SST. On the other hand, in the region East of the Azores and Portugal currents (boxes 38, 39, 41, 42, 43, 46 and 47) vertical correlations reveal a very sharp transition for both SST-reconstructed and surface-buoyancy reconstructed buoyancies (see figure 9). This suggests that the mixed layer is probably disconnected from the ocean interior, making the flow reconstruction from surface data very difficult. This could explain the poor correlations found in section 4.2. The wind distribution shown in figure 1 and the RMS of wind and mixed layer depths in figure 6 support this interpretation.

The amplitude of the reconstructed signal is analyzed, comparing the RMS of the reconstructed fields with the RMS calculated from the POP model (e.g. figure 10). The RMS of model buoyancy is characterized by a subsurface maximum value located between 100 and $300 \mathrm{~m}$ depth. In the boxes located to the East and Northeast of the studied area a second extreme at $700 \mathrm{~m}$ depth is found. Its comparison with the estimations from surface buoyancy and SST reveals that the eSQG hardly reproduces these patterns. This is because the vertical modulation of the spectral amplitude is a decaying function (see equation 10) and therefore it cannot reproduce subsurface maxima. In addition, SST tends to overestimate the RMS of buoyancy, probably because of the cancellation of density gradients due to the compensation between temperature and salinity. In practice, this effect could be suppressed by adding a new constant for buoyancy reconstruction (i.e. in front of the rhs of equation (17)). In contrast to buoyancy RMS, the eSQG approach reconstructs quite well the variations of the vorticity RMS derived from SST and surface buoyancy. The reconstruction of vorticity RMS from SSH sometimes gives values which are too small in the first hundreds of meters. As for the velocity reconstruction, the amplitude is a bit too high for the zonal velocity. Overall, the level of eddy activity (in terms of kinetic energy or enstrophy) seems qualitatively correct for all reconstructions. This means that the vertical averaged Brunt-Väisälä frequency is sufficient to determine the vertical decay scale given by $n_{0}$.

\section{Discussion}

The eSQG method relies on the assumptions that the ocean is close to QG balance and that surface buoyancy dynamics strongly affect the upper ocean dynamics. In the simulation we examined, the Rossby number (measured as the ratio of the vorticity RMS divided by the Coriolis parameter) is smaller than 0.4. Also the flow is close to geostrophic balance since the vorticity computed from SSH as streamfunction is almost identical to the observed vorticity. In more realistic simulations, for mesoscales, the Rossby number stays small whereas it can be larger than 1 for submesoscales $(<10 \mathrm{~km})$, as shown in simulation of Klein et al. [2007]. The second hypothesis is that, when inverting PV to obtain the streamfunction, the contribution of the surface buoyancy is larger than the contribution of the interior density. As shown by Lapeyre [2008] for the same simulation, this is generally true (except in the recirculating part of the gyre).

SQG theory predicts that the spectrum of surface buoyancy, or SST (if salinity is constant or correlated to it), is identical to the kinetic energy spectrum (equations (16) and (19)) and should have a spectral slope of $k^{-5 / 3}$ [Blumen, 1978]. This is confirmed for wavelengths between 10 and $400 \mathrm{~km}$ for very high resolution numerical simulations of geophysical turbulence forced by a large scale temperature gradient [Klein et al., 2007]. In addition, recent analysis of altimetric measurements has confirmed that in eddy energetic regions surface energy exhibits a $k^{-5 / 3}$ slope for wavelengths between 100 and $400 \mathrm{~km}$ [Le Traon et al., 2008]. The analysis of altimetric maps and microwave SST also shows similar spectral power laws between energy and SST spectra (although steeper) [Isern-Fontanet et al., 2006]. Finally, estimations of the SST spectra in the North Atlantic derived from remotely sensed data can exhibit power laws ranging between $k^{-1.4}$ to $k^{-2.5}$ for wavelengths between 10 to $100 \mathrm{~km}$ [Viehoff, 1989] close to the $-5 / 3$ value. This is consistent with our findings of section 4.1 where a close resemblance between surface energy spectra below $300 \mathrm{~km}$ was observed. However, the energy spectral slopes found here are steeper $\left(\sim k^{-3.8}\right)$ due to the relatively low spatial resolution of the numerical experiments performed here. As recognized by Armi and Flament [1995], the spectral slopes are not enough to characterize a physical signal. A more important quantity is the phase of this signal. The good spectral correlation between the phases of the streamfunction and the SST indicates the validity of the eSQG approach.

A possible explanation on the fact that eSQG reconstruction from SST works better than eSQG reconstruction from surface density is that SST contrasts better resemble those of the density below the mixed layer. Indeed, previous numerical studies [e.g. Klein and Hua, 1990] revealed that mixed layer deepening due to wind stress should makes the surface density constrasts to resemble subsurface fields. Here surface density contrasts seem to be much weaker than SST ones, probably because of the spatial resolution that has more impact of this field because of its steep spectrum slope. This hypothesis is confirmed in section 4.2 , where it has been shown that the linear correlation between model vorticity and SST reconstructed vorticity increases as wind increases. However, some boxes exhibit high correlations even though winds were weak. For the particular case examined here, the depth of the mixed layer seems to be a better indicator of the quality of the reconstruction, as it has been shown in 
figure 6 . When analyzing real data, the depth of the mixed layer is not known a priori, which implies that mixed layer deepening periods have to be identified using wind measurements.

The eSQG reconstruction from SST has a potential drawback because the salinity distribution is not known. Salinity anomalies can be quite large compared to temperature anomalies in the density equation, so that density anomalies can be uncoupled with SST anomalies. However, in general, salinity anomalies possess similar structures as temperature anomalies due to the advection by the geostrophic eddies that create sharp gradients of both fields in the same regions [Klein et al., 1998]. In situ observations [e.g. Ferrari and Rudnick, 2000] show a tendency for salinity and density gradients to be aligned and pointing into opposite directions. Therefore, temperature fronts would tend to be compensated by salinity fronts, as it has been observed in section 3.3. This implies that equation (17) would overestimate density gradients. We can expect that the effect of salinity can be modeled at first order as a simple modification of the Prandtl ratio. Observations reveal that at the base of the mixed layer a typical value of the quotient $|\alpha T| /|\beta S|$ is close to 2 [Ferrari and Rudnick, 2000]. Therefore, when surface fields can be considered as a proxy of the fields at the base of the mixed layer, we expect that $n_{T} \approx 2 n_{b}$, which is consistent with the values found in section 3.4.

Given these different aspects and potential weaknesses, the eSQG framework suggests an efficient method for the reconstruction of ocean dynamics from remotely sensed SST. The proposed methodology has several advantages. The method is simple and robust since the stream-function is obtained from a single image and has the same resolution as the original field (although the range of validity is between 10 to $400 \mathrm{~km}$ ). Surface fields require the determination of only one parameter $\left(n_{T}\right)$ and subsurface fields are recovered if a second parameter (the vertical average of the Brunt-Väisälä frequency $N_{0}=n_{0} f_{0}$ ) is known. A practical application of the method first requires the determination of $n_{T}$. The simplest procedure is to compare the reconstructed fields with altimetric measurements (section 3.4) as previously proposed by Isern-Fontanet et al. [2006]. The second parameter can be estimated through the in situ Argo network or a climatology of the Brunt-Väisälä frequency. The main requirement of the method is that SST has to be a proxy of the density below the mixed layer which can be approximately identified through the analysis of remotely sensed surface winds.

Presently, remote measurements of SSH and SST have very different sampling characteristics. Altimeters provide a good measure of the sea level but are strongly limited by their sampling. This makes them very well suited for the estimation of the spectral energy of the ocean but less suited for the spatial organization of surface oceanic finescale structures. On the contrary, infrared and microwave radiometers provide a precise location of ocean surface structures but the different processes that affect the measure may degrade the observed gradients and their spectral characteristics. An improved method would use complementary information given by both SST and SSH to reconstruct the stream-function.

Finally as already mentioned, the quality of the reconstruction is constrained to the upper levels of the ocean (i.e. the first 500 hundreds of meters). If the vertical variation of PV is known or modeled as in LaCasce and Mahadevan [2006], equations (5) and (8) then offer a simple way to add the necessary information and improve the reconstruction. The eSQG approach has some points in common with the method of Haines [1991] for assimilating surface data and for propagating the information at depth. This author proposed to invert a 3 layer QG model assuming a uniform PV distribution in the interior and that one knows the upperlayer streamfunction. The technique here is a bit different considering the surface buoyancy signal instead of the surface (or the upper-layer) streamfunction.

\section{Conclusions}

In this study the eSQG approach has been evaluated using a numerical OGCM simulation of the North Atlantic ocean with realistic forcings. This method allows to reconstruct the 3D dynamics of the upper ocean (the upper 500 hundreds meters) from a single snapshot of the SST field and only requires two parameters: the first one is a vertical average of the Brunt-Väisälä frequency which determines the vertical attenuation of surface fields. The second one is an effective Prandtl ratio which determines the surface kinetic energy level as a function of potential energy level. This last constant parameter parametrizes the amplitude of the contribution associated with the interior PV distribution and the effect of density compensation due to salinity distribution. Results indicate that salinity compensation has a stronger effect than the interior PV distribution.

Results also show that the strongest constraint to the applicability of the method is bounded to the dynamics of the Mixed Layer. Indeed, the success of the method depends on the quality of the SST as a proxy of the density anomaly at the base of the Mixed Layer, which happens after a Mixed Layer deepening period. Therefore, the ideal situation for the application of this method would be after strong winds. In addition, results clearly show that SST is a better proxy of density anomaly below the Mixed Layer than surface density anomaly. This is likely due to processes that force SST to resemble subsurface density whereas surface density departs from it.

The eSQG framework can further be applied to the reconstruction of subsurface fields using surface information. In this case only the mean Brunt-Väisälä frequency is required to propagate surface fields. Results have shown that the reconstruction of velocities and vorticity from surface fields are reasonably good (down to 500 meters) although the reconstruction of subsurface density anomaly is quite limited.

Finally, the technique presented in this paper may strongly contribute to some open questions in oceanography. First, it opens the door to the obtainment of synoptic high resolution velocity fields using infrared SST. Second, it provides a simple and robust dynamical framework for the merging of different data sets such as SST and SSH. Third, it has a strong potential for assimilating surface data into numerical models and propagating the mesoscale signal at depth. Clearly, further investigation needs to be addressed in these directions, in particular, to more closely focus on the role of the mixed layer.

Acknowledgments. We would like to thank P.Y. Le Traon, P. Flament and J. Ballabrera for various useful comments. Jordi Isern-Fontanet is supported by an Intra-European Marie-Curie Fellowship (MEIF-CT-2006-041476). Patrice Klein and Guillaume Lapeyre are supported by CNRS.

\section{Notes}

1. We excluded boxes 5 and 9 which have values close to $10^{\circ}$ and boxes $13,17,18$ and 21 which have values between $70^{\circ}$ and $160^{\circ}$

2. Obviously, the assumption of having surface density is unrealistic at present, but we make this assumption in order to enable comparison.

\section{References}

Afanasyev, Y., A. Kostianoy, A. Zatsepin, and P. Poulain (2002), Analysis of velocity field in the eastern Black Sea from satellite data during the Black Sea '99 experiment, J. Geophys. Res., 107, doi:10.1029/2000JC000578. 
Armi, L., and P. Flament (1995), Cautionary remarks on the spectral interpretation of turbulent flows, J. Geophys. Res., 90, 11,779-11,782.

Blumen, W. (1978), Uniform potential vorticity flow: Part I. Theory of wave interactions and two-dimensional turbulence, $J$. Atmos. Sci., 35, 774-783.

Brachet, S., P. Y. Le Traon, and C. Le Provost (2004), Mesoscale variability from a high resolution model and from altimeter data in the north atlantic ocean, J. Geophys. Res., 109, doi: 10.1029/2004JC002360.

Bretherton, F. P. (1966), Critical layer instability in baroclinic flows, Q. J. R. Meteorol. Soc., 92, 325-334.

Bryan, F. O., M. W. Hecht, and R. D. Smith (2007), Resolution convergence and sensitivity studies with North Atlantic circulation models. Part I: The western boundary current system, Ocean Modelling, 16(3-4), 141-159.

Chapron, B., F. Collard, and F. Ardhuin (2005), Direct measurements of ocean surface velocity from space: Interpretation and validation, J. Geophys. Res., 110, C07008, doi: 10.1029/2004JC002809.

de Boyer Montégut, C., G. Madec, A. Fischer, A. Lazar, and I. Iudicone (2004), Mixed layer depth over the global ocean: An examination of profile data and a profile-based climatology, $J$. Geophys. Res., 109, doi:10.1029/2004JC002378.

Emery, N. J., A. C. Thomas, M. J. Collins, W. Crawford, and D. Mackas (1986), An objective method for computing advective surface velocities from sequential infrared satellite images, J. Geophys. Res., 91, 12,865-12,878.

Ferrari, R., and F. Paparella (2003), Compensation and alignement of thermohaline gradients in the ocean mixed layer., $J$. Phys. Oceanogr., 33, 2214-2223.

Ferrari, R., and D. Rudnick (2000), Thermohaline variability in the upper ocean, J. Geophys. Res., 105, 16,857-16,883.

Haines, K. (1991), A direct method for assimilating sea surface height data into ocean models with adjustments to the deep circulation, J. Phys. Oceanogr., 21, 843-868.

Held, I., R. Pierrehumbert, S. Garner, and K. Swanson (1995), Surface quasi-geostrophic dynamics, J. Fluid Mech., 282, 120.

Hoskins, B., M. McIntyre, and R. A.W. (1985), On the use and significance of isentropic potential vorticity maps, $Q . J . R$. Meteorol. Soc., 111, 877-946.

Isern-Fontanet, A., J. Turiel, García-Ladona, and J. Font (2007), Microcanonical multifractal formalism: Application to the estimation of ocean surface velocities, J. Geophys. Res., 112, C05024, doi:10.1029/2006JC003878.

Isern-Fontanet, J., B. Chapron, P. Klein, and G. Lapeyre (2006), Potential use of microwave sst for the estimation of surface ocean currents, Geophys. Res. Lett., 33, L24608, doi: 10.1029/2006GL027801

Kelly, K. A. (1989), An inverse model fro near-surface velocity from infrared images, J. Phys. Oceanogr., 19, 1845-1864.

Klein, P., and B. L. Hua (1990), The mesoscale variability of the sea surface temperature: an analytical and numerical model., J. Mar. Res., 48, 729-763.

Klein, P., A. Tréguier, and B. L. Hua (1998), Quasigeostrophic stirring of thermohaline fronts, J. Mar. Res., 56, 589-612.

Klein, P., B. L. Hua, G. Lapeyre, X. Capet, S. Le Gentil, and H. Sasaki (2007), Upper ocean turbulence from high 3-D resolution simulations, J. Phys. Oceanogr., p. in press.
LaCasce, J., and A. Mahadevan (2006), Estimating subsurface horizontal and vertical velocities from sea surface temperature, J. Mar. Res., 64, 695-721.

Lapeyre, G. (2008), What mesoscale signal does the altimeter see? on the decomposition in baroclinic modes and the role of the surface boundary condition, J. Phys. Oceanogr., p. in revision.

Lapeyre, G., and P. Klein (2006), Dynamics of the upper oceanic layers in terms of surface quasigeostrophy theory, J. Phys. Oceanogr., 36, 165-176.

Large, W. G., J. C. McWilliams, and S. C. Doney (1994), Oceanic vertical mixing: A review and a model with a nonlocal boundary layer parameterization, Rev. Geophys., 32, 363-403.

Le Traon, P., P. Klein, B. L. Hua, and G. Dibarbourne (2008), Do altimeter wavenumber spectra agree with interior or surface quasi-geostrophic theory?, J. Phys. Oceanogr., p. in press.

Smith, R., M. Maltrud, F. Bryan, and M. Hecht (2000), Numerical simulation of the north atlantic ocean at $1 / 10^{\circ}$, J. Phys. Oceanogr., 30, 1532-1561.

Strong, A., and R. DeRycke (1973), Ocean currents monitoring using a new satellite sensing technique, Science, 182, 482-484.

Tulloch, R., and K. Smith (2006), A new theory for the atmospheric energy spectrum: Depth-limited temperature anomalies at the tropopause, P. Natl. Acad. Sci. USA, 103, 14,69014,694 .

Turiel, A., J. Isern-Fontanet, E. García-Ladona, and J. Font (2005), A multifractal method for the instantaneous evaluation of the stream-function in geophysical flows, Phys. Rev. Lett., 95, 104502.

Viehoff, T. (1989), Mesoscale variability of sea surface temperature in the North Atlantic, Int. J. Remote Sensing, 10, 771785.

Vigan, X., C. Provost, R. Bleck, and P. Courtier (2000), Sea surface velocities from sea surface temperature images sequence 1 . Method and validation using primitive equation model output, J. Geophys. Res., 105, 19,499-19,514.

Zavialov, P., J. Grigorieva, O. Moller Jr., A. Kostianoy, and M. Gregoire (2002), Continuity preserving modified cross-correlation technique., J. Geophys. Res., 107, doi: 10.1029/2001JC001.

Jordi Isern-Fontanet, Département d'Océanographie Physique et Spatiale, IFREMER, Technopole de Brest-Iroise BP 70, 29280 Plouzan, France. (jisern@ifremer.fr)

Guillaume Lapeyre, Laboratoire de Météorologie Dynamique, Ecole Normale Supérieure, 24 rue Lhomond, 75005 Paris, France. (glapeyre@lmd.ens.fr)

Patrice Klein, Département d'Océanographie Physique et Spatiale, IFREMER, Technopole de Brest-Iroise BP 70, 29280 Plouzan, France. (pklein@ifremer.fr)

Bertrand Chapron, Département d'Océanographie Physique et Spatiale, IFREMER, Technopole de Brest-Iroise BP 70, 29280 Plouzan, France. (bchapron@ifremer.fr)

Matthew W. Hecht, Computers and Computational Sciences Division, Los Alamos National Laboratory MS B296, Los Alamos, NM, 87545 USA mhecht@lanl.gov 
Table 1. Prandtl ratios: mean value within the upper $1000 \mathrm{~m}$ $\left(n_{0}\right)$, effective value derived from surface buoyancy $\left(n_{b}\right)$ and effective value derived from surface temperature $\left(n_{T}\right)$

\begin{tabular}{cccc}
\hline & $n_{0}$ & $n_{b}$ & $n_{T}$ \\
\hline 5 & 62.7 & 99.5 & 147.4 \\
9 & 61.7 & 70.3 & 99.1 \\
10 & 54.5 & 50.4 & 105.2 \\
13 & 54.3 & 49.9 & 71.5 \\
14 & 50.7 & 51.3 & 140.5 \\
17 & 53.0 & 67.6 & 92.2 \\
18 & 45.7 & 52.0 & 108.2 \\
19 & 56.9 & 26.1 & 180.7 \\
21 & 55.0 & 53.0 & 103.4 \\
22 & 47.3 & 54.2 & 164.4 \\
23 & 53.7 & 26.6 & 156.6 \\
24 & 46.6 & 108.6 & 53.2 \\
25 & 50.9 & 41.9 & 97.1 \\
26 & 42.8 & 37.1 & 107.6 \\
27 & 42.2 & 22.5 & 130.8 \\
28 & 38.3 & 38.4 & 98.0 \\
29 & 51.0 & 43.2 & 103.0 \\
30 & 40.6 & 37.4 & 100.0 \\
31 & 37.9 & 24.2 & 95.0 \\
32 & 33.0 & 30.1 & 101.6 \\
33 & 50.9 & 40.6 & 124.9 \\
34 & 41.8 & 35.1 & 78.6 \\
35 & 33.1 & 35.5 & 88.6 \\
36 & 29.2 & 23.9 & 90.1 \\
37 & 49.4 & 41.3 & 120.0 \\
38 & 42.8 & 29.3 & 93.1 \\
39 & 31.7 & 23.0 & 62.7 \\
40 & 25.4 & 27.8 & 69.7 \\
41 & 52.2 & 58.4 & 117.3 \\
42 & 41.4 & 33.0 & 102.8 \\
43 & 33.7 & 27.5 & 76.3 \\
44 & 27.1 & 28.7 & 64.4 \\
46 & 45.8 & 45.6 & 154.4 \\
47 & 35.2 & 63.3 & 135.9 \\
48 & 24.6 & 35.7 & 74.5 \\
\hline & & &
\end{tabular}



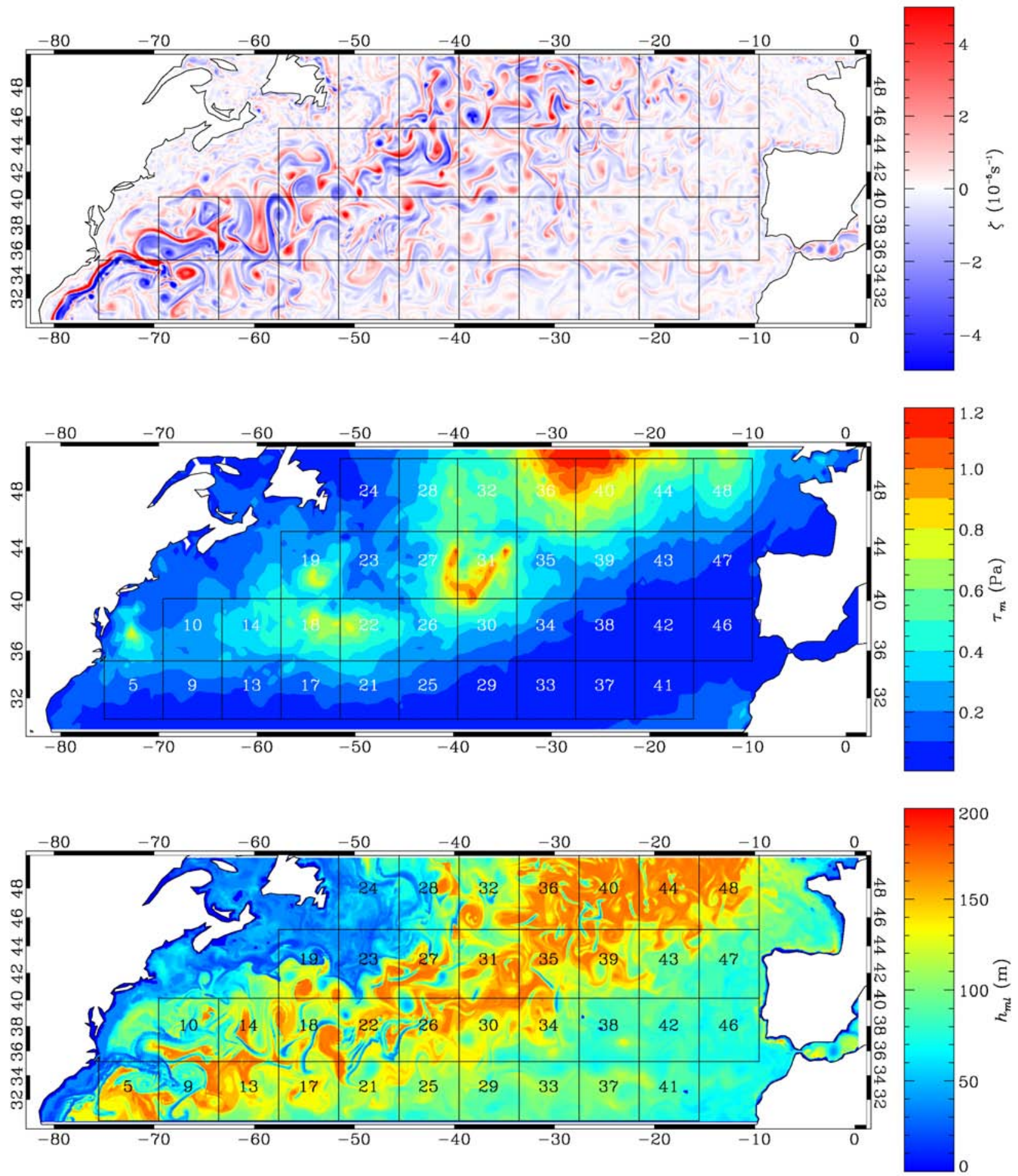

Figure 1. From top to bottom: surface vorticity $\left(\zeta_{s}\right)$, maximum wind stress observed during the preceding 2 days $\left(\tau_{m}\right)$ and mixed layer depth $\left(h_{m l}\right)$. In each box its identification number has been written. 

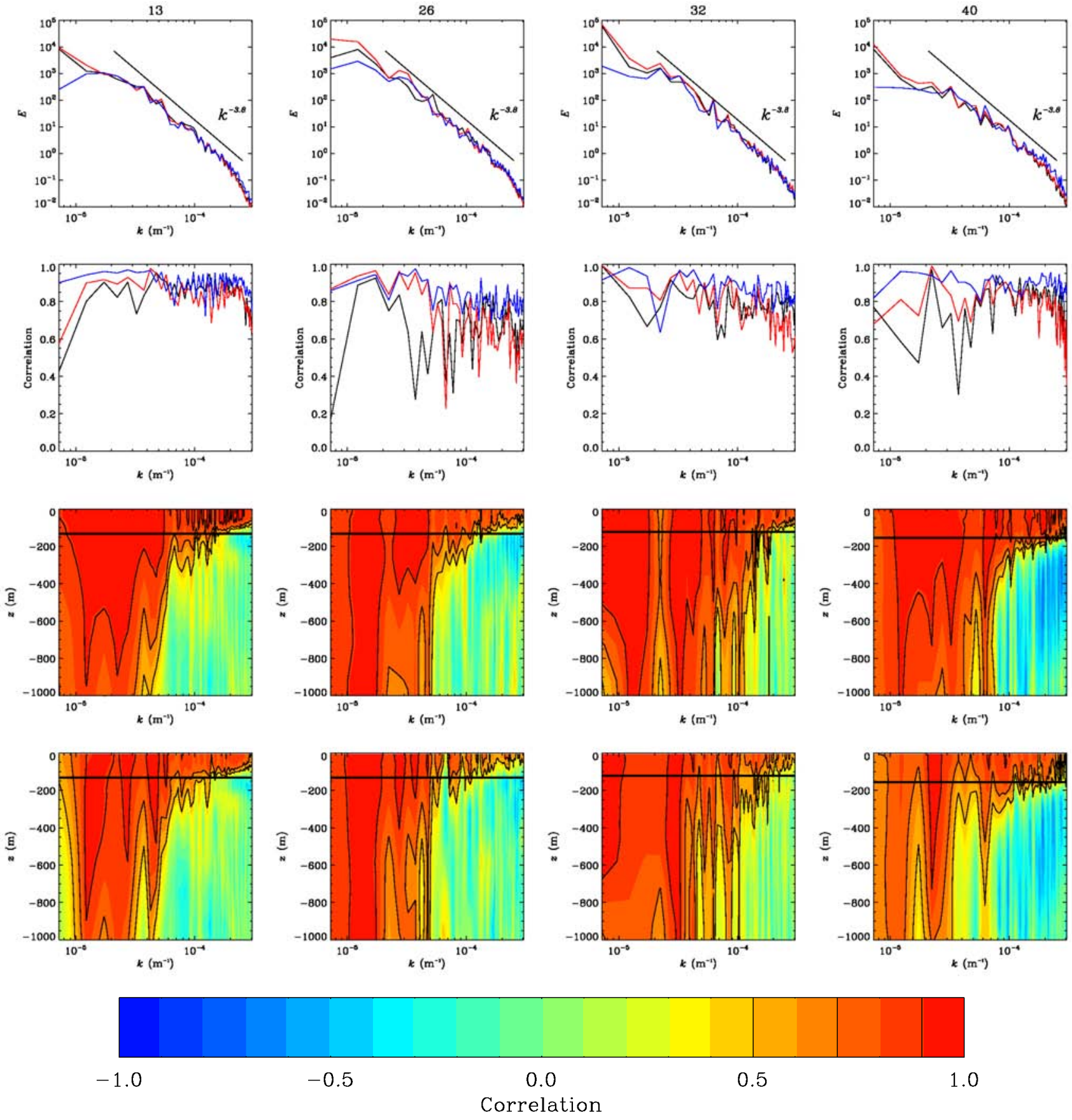

Figure 2. Top, comparison between the energy spectrum derived from the SSH (blue), from surface buoyancy (black) and from SST (red). Second row, spectral correlations between relative vorticity from the model and the vorticity derived from the SSH (blue), from surface buoyancy (black) and from SST (red) at the ocean surface. Third row, spectral correlations between surface vorticity derived from SSH and vorticity derived from velocity at different depths. Bottom, same as the third row but using surface vorticity derived from SST. Columns correspond to boxes $13,26,32$, and 40 . The thick black line in the third and fourth rows indicates the mean mixed layer depth. 

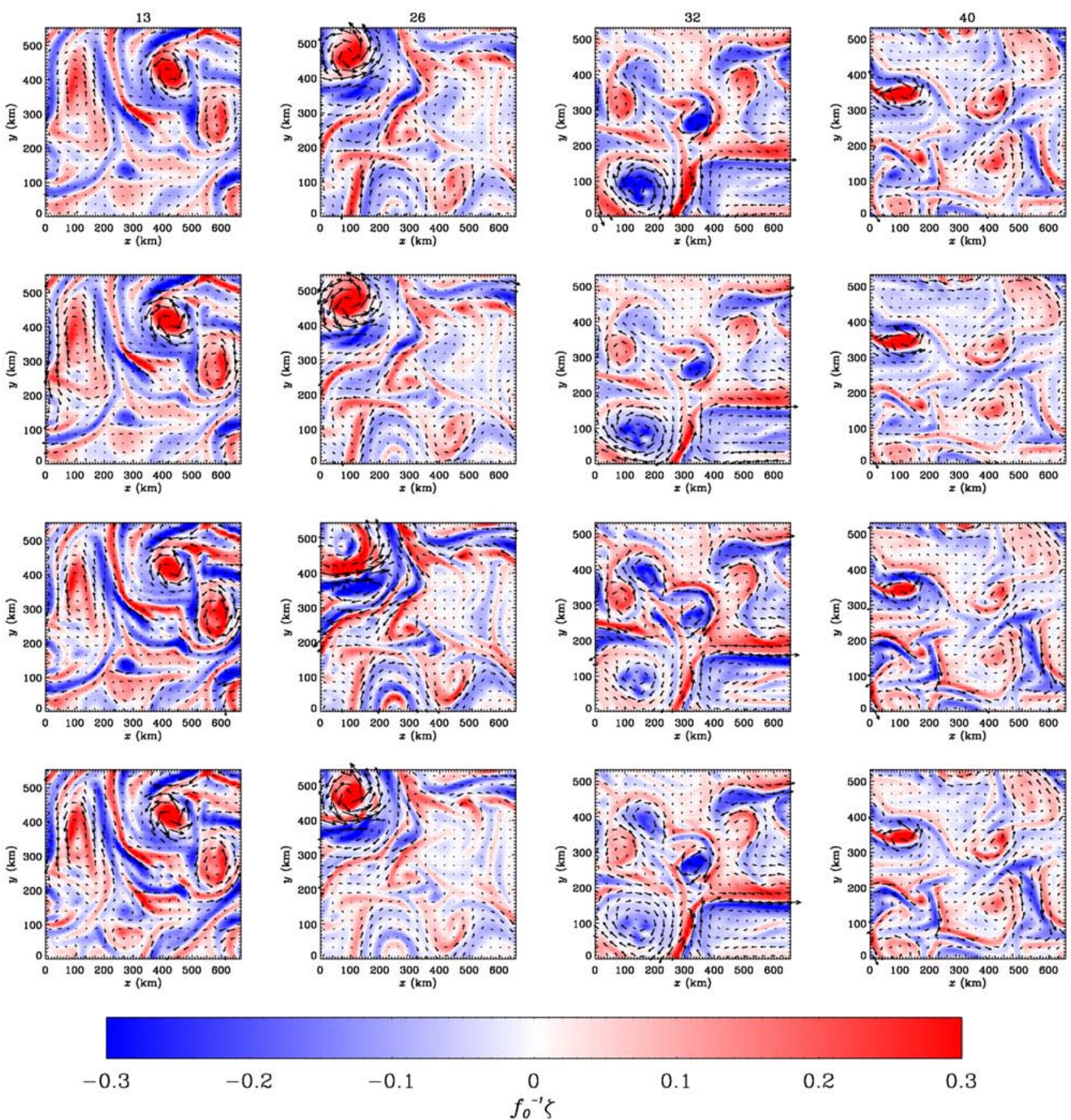

Figure 3. High-pass filtered $(\lambda \leq 350 \mathrm{~km})$ surface vorticity derived from model (first row), from SSH (second row), from surface buoyancy (third row) and from SST (fourth row). Plots correspond to boxes 13, 26, 32, and 40. Vector lengths are such that a vector with a speed of $50 \mathrm{~cm} \mathrm{~s}^{-1}$ has a length equal to vector separation. Only one over nine velocity vectors have been drawn. 

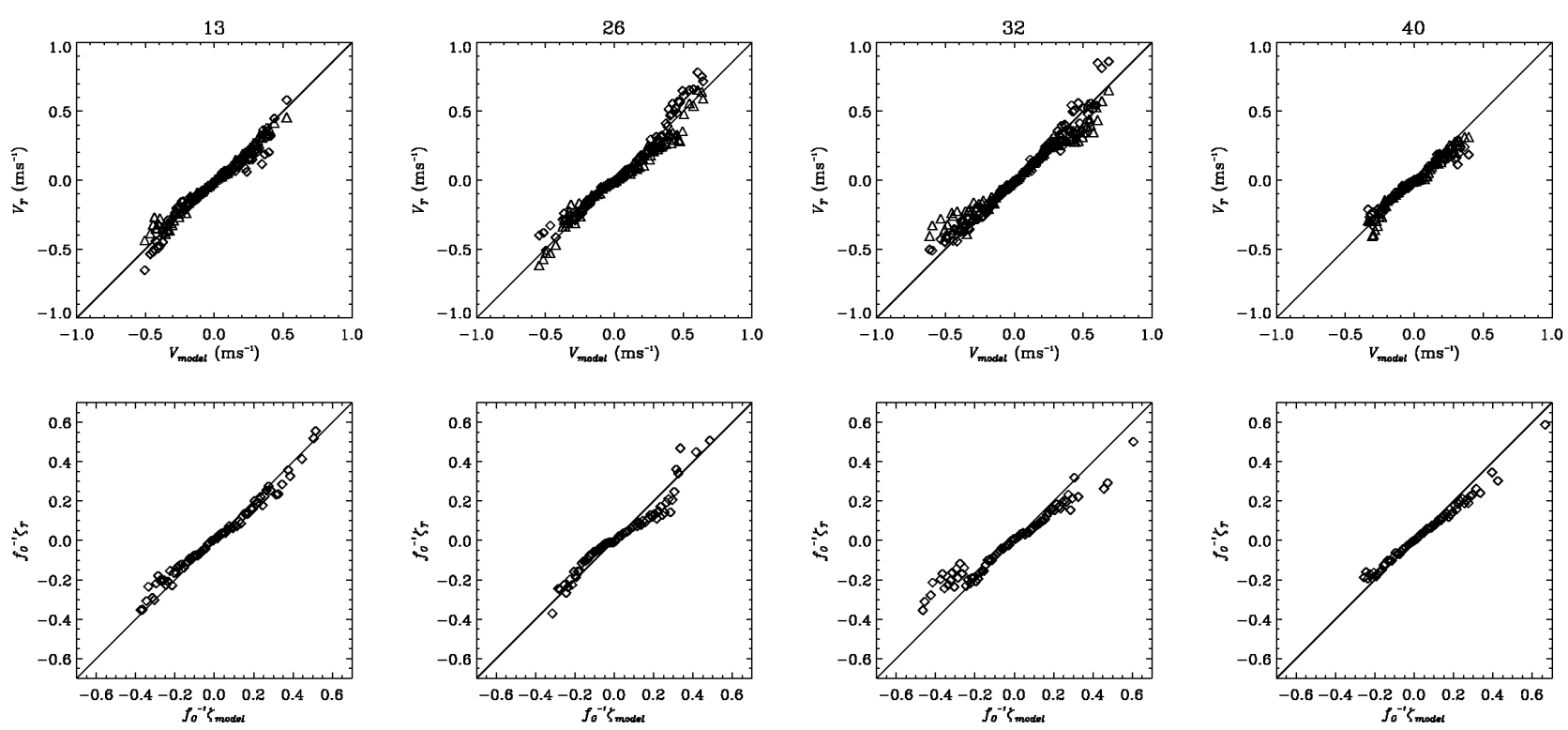

Figure 4. Scatter plot between surface velocities (top) and surface vorticities (bottom) of the model ( $\mathrm{v}_{\text {model }}$, $\left.\zeta_{\text {model }}\right)$ and their reconstruction from $\operatorname{SST}\left(\mathrm{v}_{T}, \zeta_{T}\right)$ corresponding to boxes $13,26,32$ and 40 . When comparing velocities diamonds correspond to zonal velocities and triangles to meridional velocities.
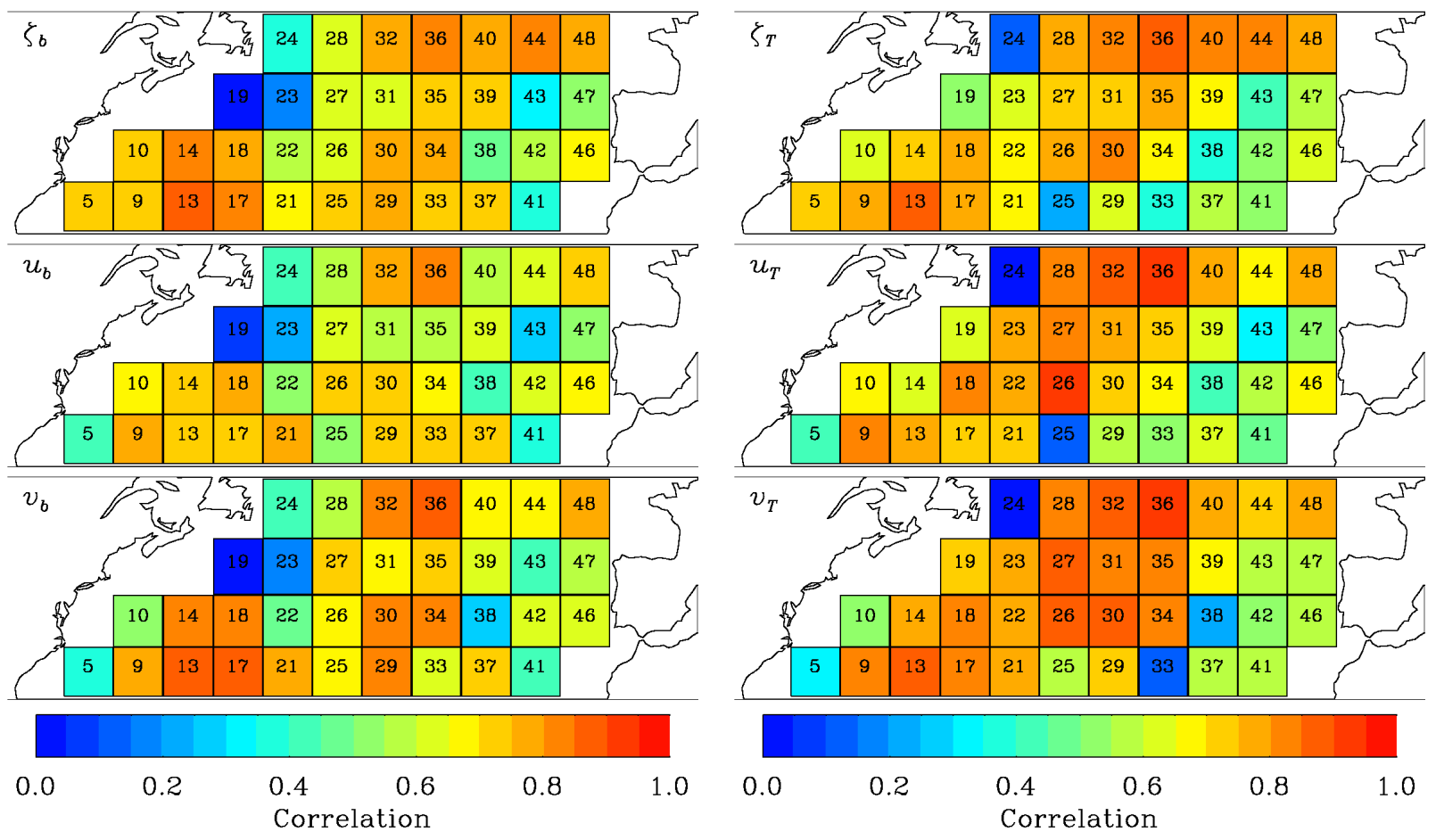

Figure 5. Linear correlation coefficients between surface vorticity and velocities from model $\left(\zeta_{s}, u_{s}, v_{s}\right)$ and the estimates obtained from surface buoyancy $\left(\zeta_{b}, u_{b}, v_{b}\right)$ and $\operatorname{SST}\left(\zeta_{T}, u_{T}, v_{T}\right)$. In each box its identification number has been written. 

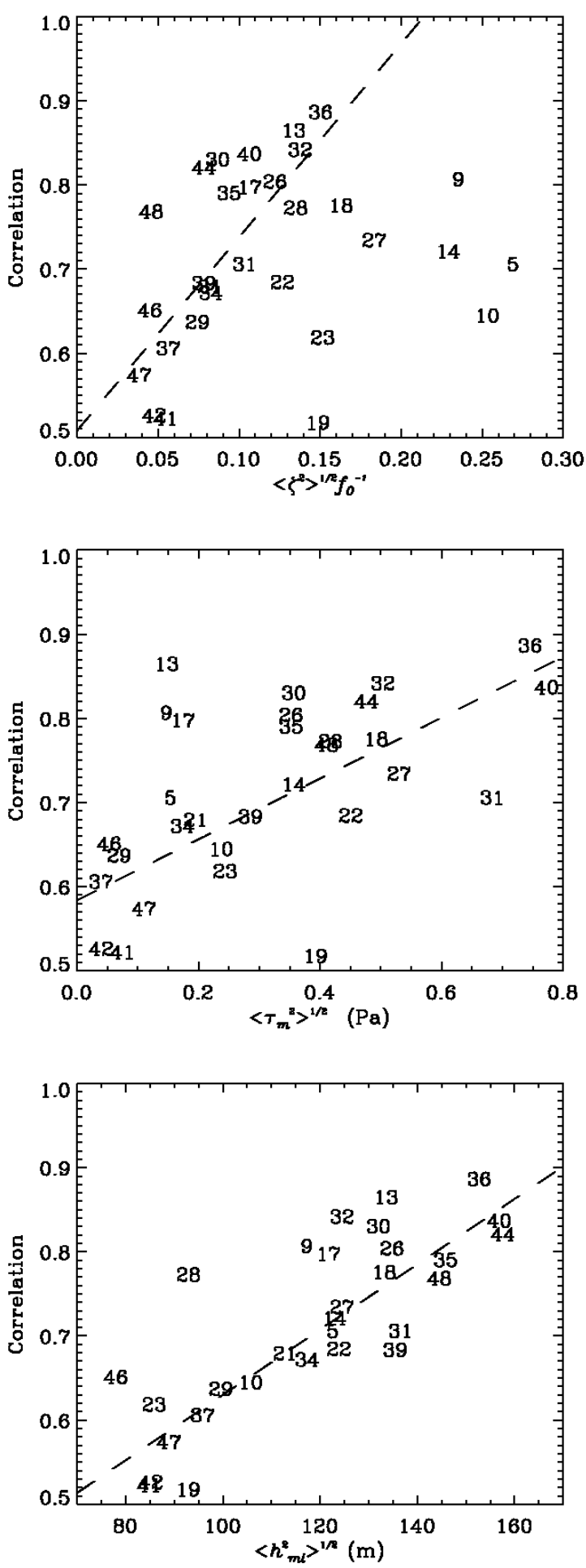

Figure 6. Scatter plots between the correlation of surface vorticity from the model and the estimate of surface vorticity derived from SST and the RMS of vorticity (top), the RMS of wind stress (middle) and the RMS of the mixed layer depth (bottom). Dashed lines correspond to a linear fit between observations. In the first plot, boxes with values of vorticity RMS above 0.15 have not been used to compute the linear fit; in the middle plot boxes $9,13,17$ and 19 have not been used; in the bottom plot box 28 has not been used. Numbers correspond to the box identification number. 

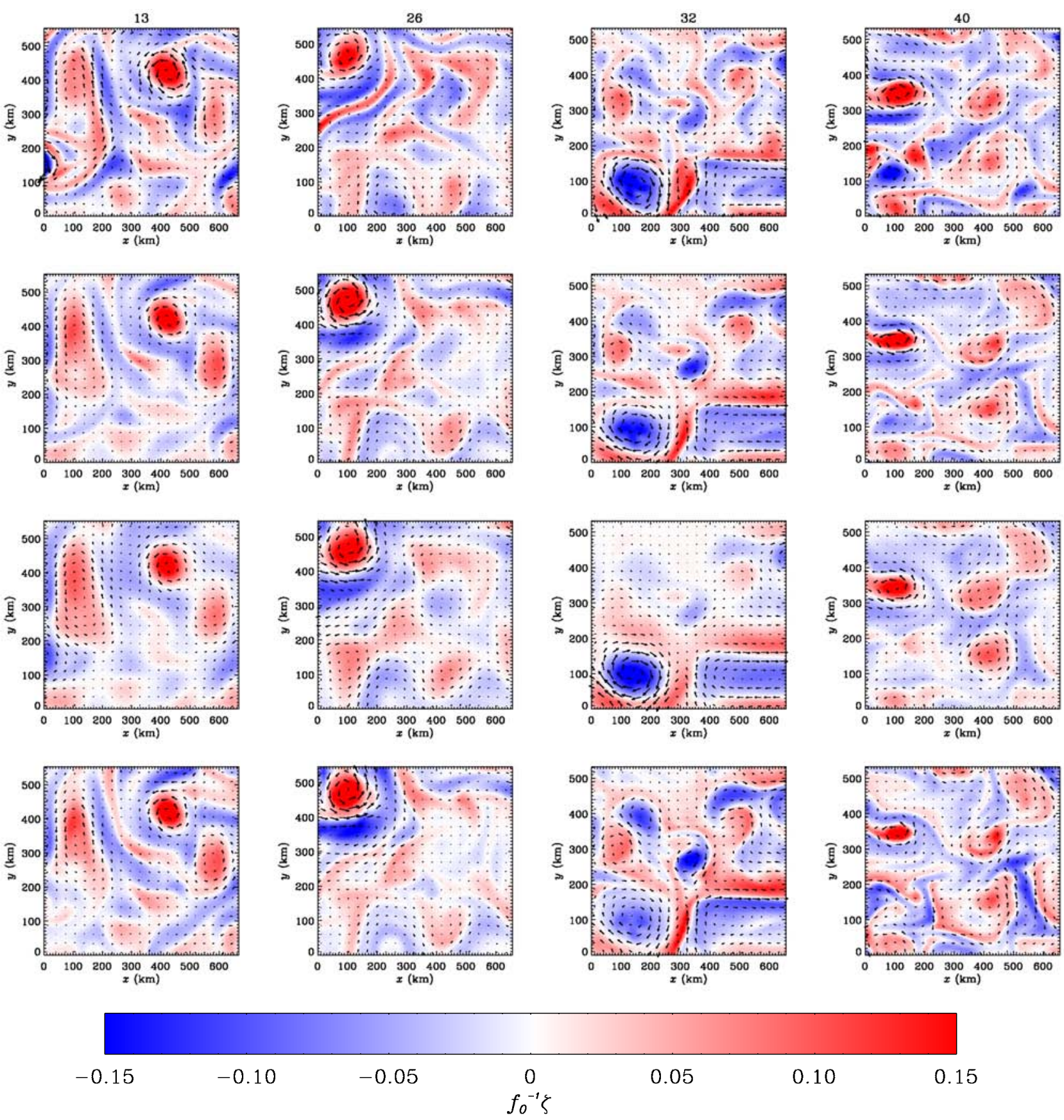

Figure 7. High-pass filtered $(\lambda \leq 350 \mathrm{~km})$ vorticity derived from velocity at $500 \mathrm{~m}$ (first row), from SSH (second row), from surface buoyancy (third row) and from SST (fourth row). Plots correspond to boxes 13, 26, 32, and 40. Vector lengths are such that a vector with a speed of $50 \mathrm{~cm} \mathrm{~s}^{-1}$ has a length equal to vector separation. Only one over nine velocity vectors have been drawn. 

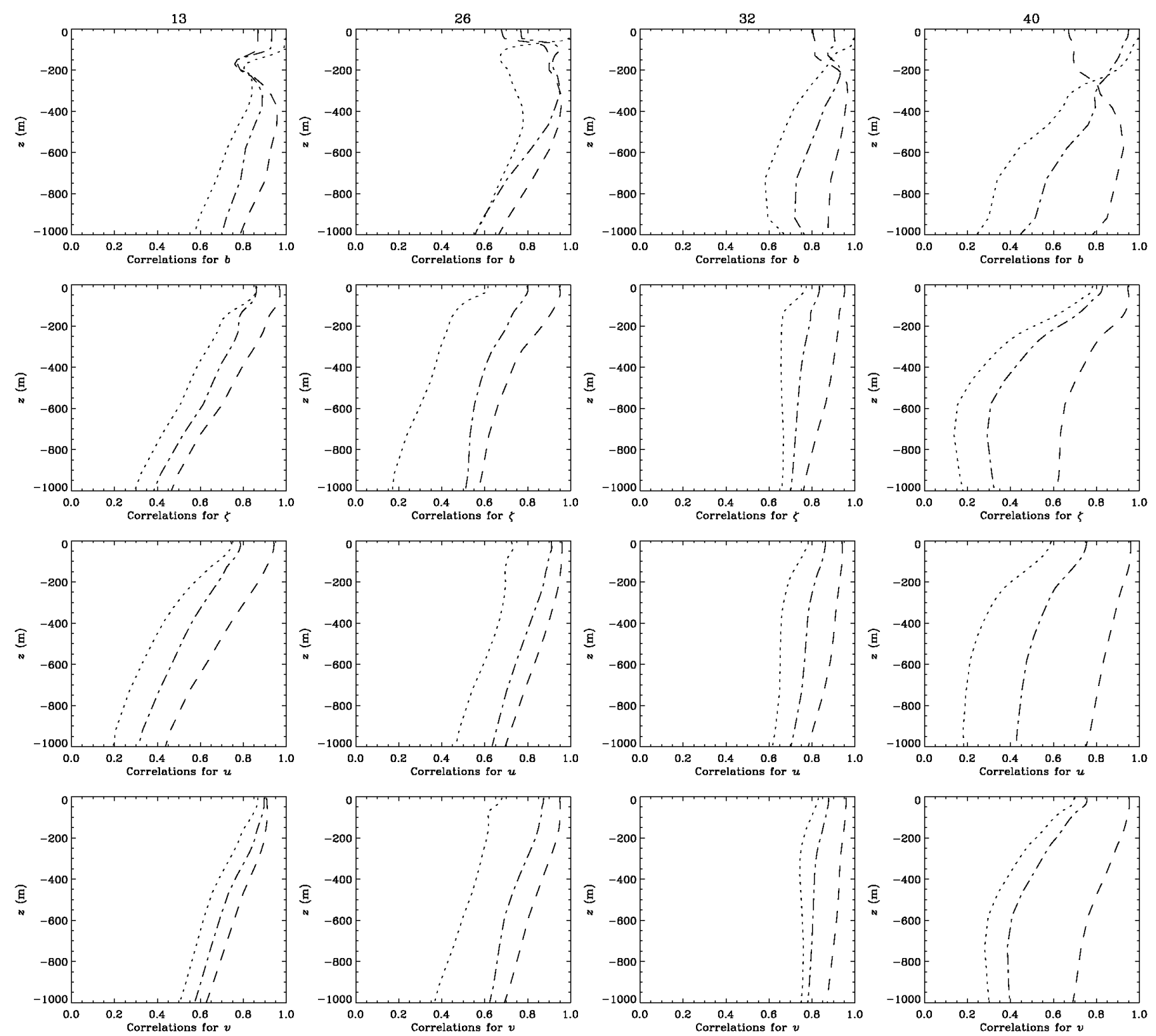

Figure 8. Vertical correlations between the fields of the model and the eSQG reconstruction from altimetry (dashed line), from SST (dash-dotted line) and from surface buoyancy (dotted line). From top to bottom: buoyancy $(b)$, vorticity $(\zeta)$, zonal velocity $(u)$ and meridional velocity $(v)$ for boxes $13,26,32$, and 40 .
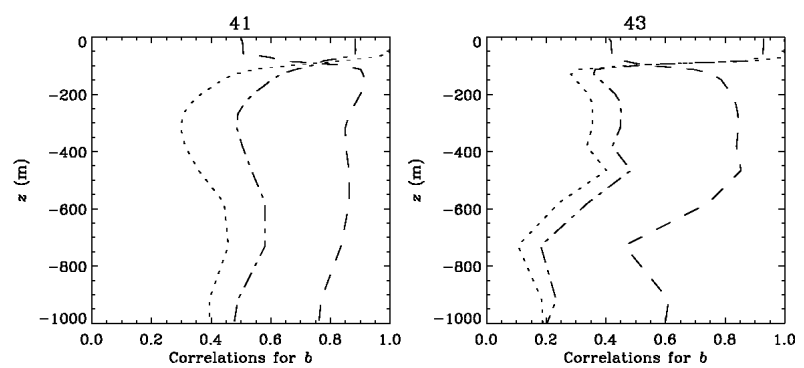

Figure 9. Vertical correlations between buoyancy of the model and the eSQG reconstruction of buoyancy from altimetry (dashed line), from SST (dash-dotted line) and from surface buoyancy (dotted line) for some boxes located east of the Azores Islands (boxes 41 and 43). 

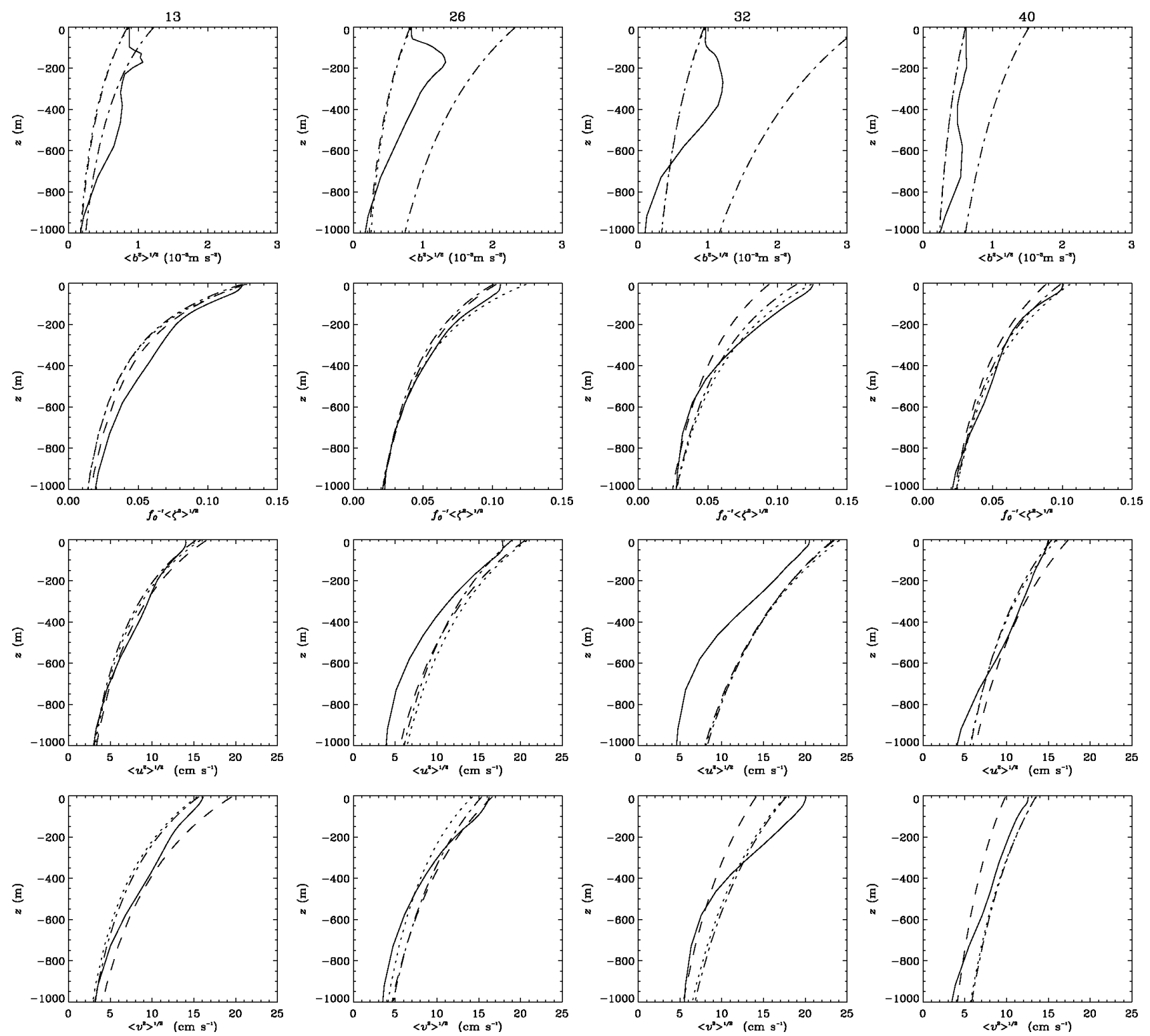

Figure 10. Vertical profiles of RMS for the fields of the model (solid line) and the eSQG reconstruction from altimetry (dashed line), from SST (dash-dotted line) and from surface density (dotted line). From top to bottom: buoyancy $(b)$, vorticity $(\zeta)$, zonal velocity $(u)$ and meridional velocity $(v)$ for boxes $13,26,32$, and 40 . 